\title{
The Clinicopathological and Prognostic Significance of the Gross Classification of Hepatocellular Carcinoma
}

Yangkyu Lee $\cdot$ Hyunjin Park ${ }^{1}$ Hyejung Lee ${ }^{1} \cdot$ Jai Young Cho $^{2}$ Yoo-Seok Yoon ${ }^{2}$. Young-Rok Choi ${ }^{2}$ Ho-Seong $\mathrm{Han}^{2}$. Eun Sun Jang ${ }^{3}$ Jin-Wook $\mathrm{Kim}^{3}$ · Sook-Hyang Jeong ${ }^{3}$ Soomin Ahn · Haeryoung Kim ${ }^{1}$

Department of Pathology, Seoul National University Bundang Hospital, Seoul National University College of Medicine, Seongnam; 'Department of Pathology, Seoul National University Hospital, Seoul National University College of Medicine, Seoul; Departments of ${ }^{2}$ Surgery and ${ }^{3}$ Internal Medicine, Seoul National University Bundang Hospital, Seoul National University College of Medicine, Seongnam, Korea

Received: August 21, 2017

Revised: October 13, 2017

Accepted: November 12, 2017

Corresponding Author

Haeryoung Kim, MD, PhD

Department of Pathology, Seoul National University

Hospital, Seoul National University College of

Medicine, 103 Daehak-ro, Jongno-gu, Seoul

03080, Korea

Tel: $+82-2-740-8322$

Fax: $+82-2-765-5600$

E-mail: haeryoung.kim@snu.ac.kr

\begin{abstract}
Background: We aimed to determine the clinicopathological significance of the gross classification of hepatocellular carcinoma (HCC) according to the Korean Liver Cancer Association (KLCA) guidelines. Methods: A retrospective analysis was performed on 242 cases of consecutively resected solitary primary HCC between 2003 and 2012 at Seoul National University Bundang Hospital. The gross classification (vaguely nodular [VN], expanding nodular [EN], multinodular confluent [MC], nodular with perinodular extension [NP], and infiltrative [INF]) was reviewed for all cases, and were correlated with various clinicopathological features and the expression status of "stemness"-related (cytokeratin 19 [CK19], epithelial cell adhesion molecule [EpCAM]), and epithelial-mesenchymal transition (EMT)-related (urokinase plasminogen activator receptor [UPAR] and Ezrin) markers. Results: Significant differences were seen in overall survival $(p=.015)$ and disease-free survival $(p=.034)$ according to the gross classification; INF type showed the worst prognosis while $\mathrm{VN}$ and EN types were more favorable. When the gross types were simplified into two groups, type 2 HCCs (MC/NP/INF) were more frequently larger and poorly differentiated, and showed more frequent microvascular and portal venous invasion, intratumoral fibrous stroma and higher $\mathrm{pT}$ stages compared to type $1 \mathrm{HCCs}$ (EN/VN) ( $p<.05$, all). CK19, EpCAM, uPAR, and ezrin expression was more frequently seen in type $2 \mathrm{HCCs}(\mathrm{p}<.05$, all). Gross classification was an independent predictor of both overall and disease-free survival by multivariate analysis (overall survival: $p=.030$; hazard ratio, $4.118 ; 95 \%$ confidence interval, 1.142 to 14.844 ; disease-free survival: $p=.016$; hazard ratio, $1.617 ; 95 \%$ confidence interval, 1.092 to 2.394$)$. Conclusions: The gross classification of HCC had significant prognostic value and type 2 HCCs were associated with clinicopathological features of aggressive behavior, increased expression of "stemness"and EMT-related markers, and decreased survival.
\end{abstract}

Key Words: Carcinoma, hepatocellular; Gross classification; Prognosis
Hepatocellular carcinomas (HCCs) vary in their macroscopic appearances, from well-circumscribed expansile nodules to those with multinodular features and irregular margins; however, the gross classification of HCC has not received much attention in the literature. Although the macroscopic classification was first described by Eggel in 1901 based on an autopsy series, it was not until the late 1980's that the gross classification was proposed and adopted by the Liver Cancer Study Group of Japan (LCSGJ) in the General Rules of the Clinical and Pathologic Study of Primary Liver Cancer. ${ }^{1,2}$ Since then, there have been several clinicopathological studies that focused on the gross features of HCC, mainly from Asian countries, and most studies have demonstrated that HCCs with single nodular morphology have more favorable outcomes compared with those with multinodular or infiltrative (INF) growth patterns. ${ }^{3-11}$ As the gross appearance of HCCs can be recognized preoperatively by imaging studies, it could have translational impact on clinical practice, such as guiding treatment decisions. However, the gross appearance was only reflected in the Cancer of the Liver Italian Program (CLIP) score (uninodular versus multinodular) which is not deemed suitable for the current population of HCC patients, ${ }^{12}$ and the current widely used staging systems, such as the modified Union for International Cancer Control (UICC), Barcelona Clinic Liver Cancer (BCLC), and American Joint Committee on Cancer 
(AJCC) TNM classifications focus on the multiplicity, size and vascular/bile duct invasion status of the HCCs.

In this study, we analyzed the differences in the clinicopathological features and survival between the different gross morphological types of HCC in a single cohort of surgically resected solitary HCCs. The gross morphology was classified according to The General Rules for the Study of Primary Liver Cancer published by the Korean Liver Cancer Association (KLCA). ${ }^{13}$

\section{MATERIALS AND METHODS}

\section{Patient selection and clinicopathological analysis}

Two hundred and ninety-eight consecutive cases of primary HCCs that were surgically resected between 2003 and 2012 at Seoul National University Bundang Hospital, Seongnam, Republic of Korea were evaluated in this study. This study was approved by the Institutional Review Board of Seoul National University Bundang Hospital (IRB No. B-1708-412-304), and patient consent was waived due to the retrospective nature of this study. Clinicopathological data were analyzed by reviewing electronic medical records, pathology reports and glass slides, and included patient sex, age at operation, tumor size, gross type, histologic differentiation (Edmondson-Steiner grade), serum $\alpha$-fetoprotein (AFP) levels and the pathological $\mathrm{T}$ and $\mathrm{N}$ categories according to AJCC TNM staging system (7th edition). The presence of intratumoral fibrous stroma was also noted; we defined the presence of intratumoral fibrous stroma as fibrous stroma occupying more than $30 \%$ of the tumor area. ${ }^{14}$ Cases with multiple HCCs were excluded from the study, leaving a total of 242 cases for further analysis. Follow-up data was retrieved from the electronic medical records, including the status at last follow up and occurrence of distant or intrahepatic metastasis or local recurrence.

The gross type was determined by examining the largest cross section of the tumor by two pathologists (Y.L. and H.K.), according to the General Rules for the Study of Primary Liver Cancer by the KLCA (Fig. 1). ${ }^{13}$ "Vaguely nodular" (VN) type was defined as a nodule with indistinct margins. While VN type morphology is an important characteristic of early $\mathrm{HCC},{ }^{15}$ we classified HCCs as VN type purely by gross appearance, regardless of the tumor size and histologic differentiation status. "Expanding nodular" (EN) type was defined by as a round expansile nodule with a distinct margin. "Multinodular confluent" (MC) type was defined as a cluster of small and confluent nodules. "Nodular with perinodular extension" (NP) type was defined as an expanding
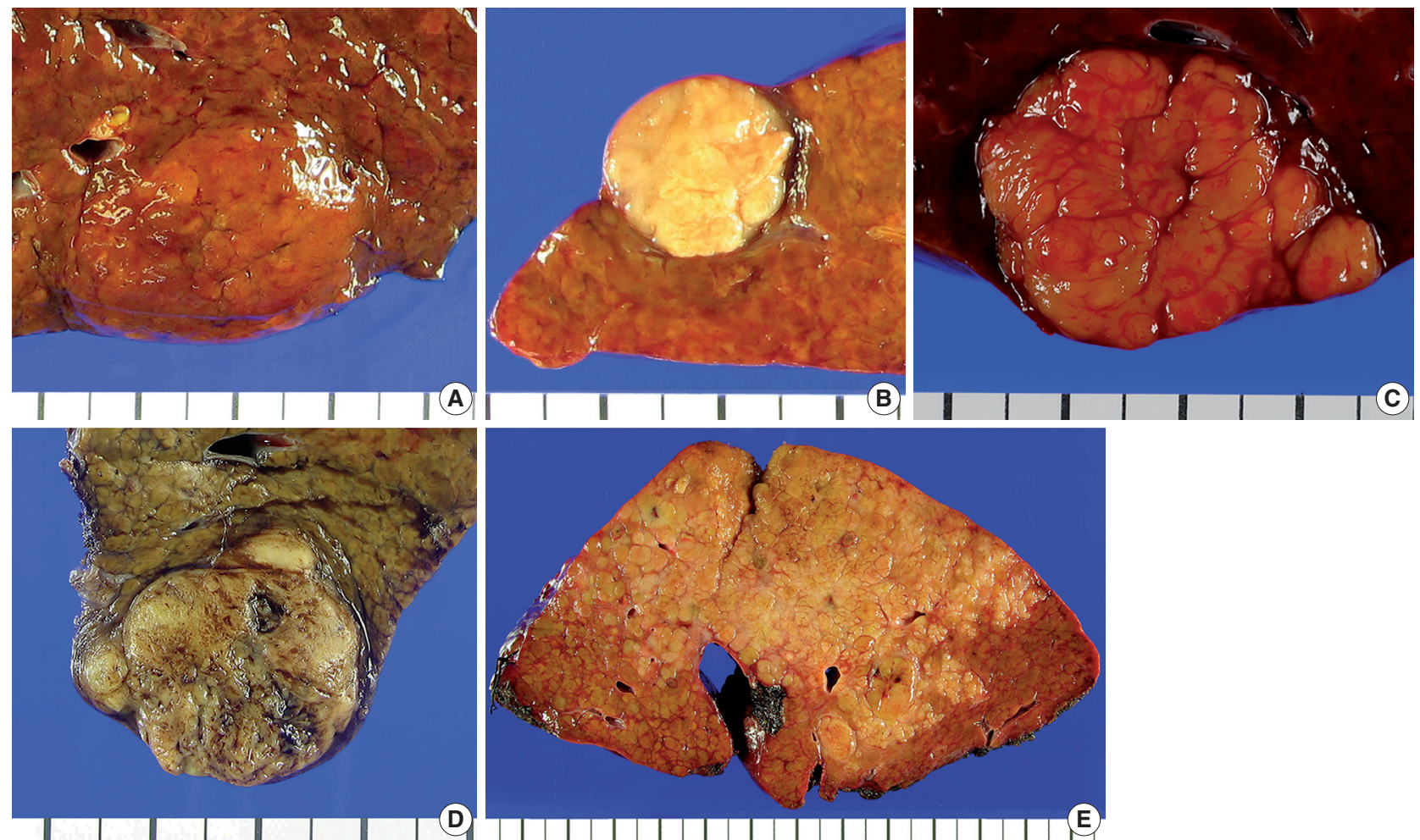

Fig. 1. Examples of the different gross types of hepatocellular carcinoma. (A) Vaguely nodular type. (B) Expanding nodular type. (C) Multinodular confluent type. (D) Nodular with perinodular extension type. (E) Infiltrative type. 
nodule similar to EN type HCCs that had extranodular growth in less than $50 \%$ of the tumor circumference. INF type HCCs showed extranodular growth in more than $50 \%$ of the tumor circumference. The gross types were correlated with the clinicopathological features of the HCCs.

\section{Tissue microarray construction and immunohistochemistry}

Two-millimeter-core tissue microarrays were constructed from the HCCs (Superbiochips Laboratories, Seoul, Korea), and $4 \mu \mathrm{m}$ thick tissue sections obtained from the tissue microarray blocks. Immunohistochemical staining was performed for "stemness"related markers (cytokeratin 19 [CK19]; 1:100, mouse monoclonal, Dako, Glostrup, Denmark), epithelial cell adhesion molecule (EpCAM; 1:3,000, mouse monoclonal, Millipore, Billerica, MA, USA), and epithelial-mesenchymal transition (EMT)-related markers (urokinase plasminogen activator receptor [UPAR; 1:40, mouse monoclonal, Abcam, Cambridge, UK] and ezrin [1:100, mouse monoclonal, Abcam]). Briefly, after deparaffinization in xylene and rehydration in graded alcohol, antigen retrieval was performed on tissue sections using citrate buffer $(\mathrm{pH}$ 6.0) for CK19, EpCAM, and ezrin, and protease for uPAR. Incubation with primary antibodies was performed for 1 hour at room temperature, and with secondary antibody (EnVision kit, Dako) for 30 minutes. The presence of cytoplasmic expression in $>5 \%$ of the tumor cells was regarded as positive for CK19, ezrin, and UPAR expression. EpCAM was expressed in the tumor cell membranes.

\section{Statistical analysis}

All statistical analyses were performed using SPSS ver. $19.0 \mathrm{~K}$ (SPSS Korea, Seoul, Korea). Chi-square tests and Fisher exact tests were performed as deemed appropriate. Survival analyses for overall and disease-free survivals were performed by the KaplanMeier method and log-rank test. The Cox regression models were used for multivariate analysis. Statistical significance was defined as $\mathrm{p}<.05$.

\section{RESULTS}

\section{Clinicopathological characteristics according to HCC gross classification}

The clinicopathological characteristics of the 242 cases studied are summarized in Table 1. The most common etiologic factor was hepatitis B virus (HBV) infection (171/242, 70.7\%), followed by alcohol $(35 / 242,14.5 \%)$, hepatitis $\mathrm{C}$ virus ( $\mathrm{HCV}$ ) infection $(17 / 242,7.0 \%)$ and combined HBV + HCV infection $(1 / 242,0.4 \%)$. The etiology was uncertain for the remainder of
Table 1. Summary of the clinicopathological characteristics

\begin{tabular}{|c|c|}
\hline Characteristic & No. $(\%)(n=242)$ \\
\hline \multicolumn{2}{|l|}{ Sex } \\
\hline Male & $181(74.8)$ \\
\hline Female & $61(25.2)$ \\
\hline Age at operation, median (range, yr) & $59(29-87)$ \\
\hline Preoperative serum AFP level, median (range, ng/mL) & $14.35(1-40,000)$ \\
\hline \multicolumn{2}{|l|}{ Etiology } \\
\hline Hepatitis B virus (HBV) & $171(70.7)$ \\
\hline Alcohol & $35(14.5)$ \\
\hline Hepatitis C virus (HCV) & $17(7.0)$ \\
\hline $\mathrm{HBV}+\mathrm{HCV}$ & $1(0.4)$ \\
\hline Uncertain etiology & $18(7.4)$ \\
\hline Tumor size, median (range, cm) & $3.0(0.9-17.0)$ \\
\hline$\leq 2$ & $52(21.5)$ \\
\hline$>2$ and $\leq 5$ & $145(59.9)$ \\
\hline$>5$ & 45 (18.6) \\
\hline \multicolumn{2}{|l|}{ Gross type } \\
\hline Vaguely nodular & $9(3.7)$ \\
\hline Expanding nodular & $107(44.2)$ \\
\hline Multinodular confluent & 78 (32.2) \\
\hline Nodular with perinodular extension & $32(13.2)$ \\
\hline Infiltrative & $16(6.6)$ \\
\hline \multicolumn{2}{|l|}{ Edmondson-Steiner grade } \\
\hline Grade I & $2(0.8)$ \\
\hline Grade II & $74(30.6)$ \\
\hline Grade III & $142(58.7)$ \\
\hline Grade IV & $24(9.9)$ \\
\hline \multicolumn{2}{|l|}{ Microvascular invasion } \\
\hline Absent & $154(63.6)$ \\
\hline Present & $88(36.4)$ \\
\hline \multicolumn{2}{|l|}{ Portal vein invasion } \\
\hline Absent & $225(93.0)$ \\
\hline Present & $17(7.0)$ \\
\hline \multicolumn{2}{|l|}{ Cirrhosis in background liver } \\
\hline Absent & $109(45.0)$ \\
\hline Present & $126(52.1)$ \\
\hline \multicolumn{2}{|l|}{ Intratumoral fibrous stroma (>30\%) } \\
\hline Absent & $187(77.3)$ \\
\hline Present & $55(22.7)$ \\
\hline \multicolumn{2}{|l|}{ Pathologic T category (AJCC 7th edition) } \\
\hline pT1 & $140(57.9)$ \\
\hline pT2 & $82(33.9)$ \\
\hline pT3 & $17(7.0)$ \\
\hline pT4 & $3(1.2)$ \\
\hline \multicolumn{2}{|l|}{ Pathologic N category (AJCC 7th edition) } \\
\hline pNO & $240(99.2)$ \\
\hline pN1 & $2(0.8)$ \\
\hline \multicolumn{2}{|l|}{ Recurrence on follow-up } \\
\hline Absent & $138(57.0)$ \\
\hline Present & $104(43.0)$ \\
\hline \multicolumn{2}{|l|}{ Status at last follow-up } \\
\hline Alive & $160(66.1)$ \\
\hline Deceased of disease & $19(7.9)$ \\
\hline Deceased of other cause & $5(2.1)$ \\
\hline Follow-up loss & $58(24.0)$ \\
\hline
\end{tabular}

AFP, $\alpha$-fetoprotein; AJCC, American Joint Committee on Cancer. 
patients $(18 / 298,7.4 \%)$. The most common gross type of HCCs was the EN type (107/242, 44.2\%), followed by the MC type (78/242, 32.2\%), NP type (32/242, 13.2\%), INF type (16/242, $6.6 \%)$, and the VN type $(9 / 242,3.7 \%)$.

When the clinicopathological features were compared among the five different gross types, we found that INF type HCCs were associated with larger tumor size, poor histologic differentiation, more frequent microvascular and portal venous invasion and higher pathologic $\mathrm{T}$ stage compared to the other gross types (Table 2). Large tumor size, microvascular and portal venous invasion and high $\mathrm{T}$ stage were rare or absent in $\mathrm{VN}$ or EN type HCCs. The clinicopathological features of MC and NP type HCCs were similar. We regrouped the five gross types into type 1 and type 2, as previously described by Gong et al. ${ }^{5}$ : type 1 HCCs consisted of VN and EN type, and type 2 HCCs consisted of MC, NP, and INF types. On comparing the clinicopathological findings between the two types, we found that type 2 HCCs were more frequently larger $(\mathrm{p}<.001)$ and poorly differentiated $(\mathrm{p}=$
$.001)$, and showed more frequent microvascular invasion ( $\mathrm{p}<$ $.001)$, portal venous invasion $(\mathrm{p}<.001)$, higher $\mathrm{pT}$ stages $(\mathrm{p}<$ .001 ), and intratumoral fibrous stroma $(\mathrm{p}<.001)$ compared to type 1 HCCs.

\section{Differences in expression status of "stemness"- and EMT- related markers in $\mathrm{HCC}$ according to gross classification}

The immunohistochemical stain results are summarized in Table 2 and Fig. 2. The expression of "stemness"-related markers, CK19 and EpCAM, was seen in $18.6 \%$ and $43.4 \%$ of HCCs, respectively. CK19 and EpCAM expression rates were significantly higher in INF type HCCs compared to EN type HCCs (CK19, 37.5\% vs 9.3\%; EpCAM, $75.0 \%$ vs 34.6\%). Significant differences were seen in the frequencies of $\mathrm{CK} 19$ and $\mathrm{EpCAM}$ positivity between type 1 and type 2 HCCs (CK19, $\mathrm{p}=.002$; EpCAM, $\mathrm{p}=.009)$. uPAR and ezrin, EMT-related markers, were more frequently expressed in type 2 HCCs compared to type 1 HCCs (uPAR, $\mathrm{p}<.001$; ezrin, $\mathrm{p}=.036$ ).

Table 2. Clinicopathological features and immunohistochemical stain results according to gross type

\begin{tabular}{|c|c|c|c|c|c|c|c|c|}
\hline & $\begin{array}{c}\mathrm{VN} \\
(n=9)\end{array}$ & $\begin{array}{c}\text { EN } \\
(n=107)\end{array}$ & $\begin{array}{c}M C \\
(n=78)\end{array}$ & $\begin{array}{c}N P \\
(n=32)\end{array}$ & $\begin{array}{c}\text { INF } \\
(n=16)\end{array}$ & $\begin{array}{l}\text { Type } 1 \\
\text { (VN, EN) } \\
(n=116)\end{array}$ & $\begin{array}{c}\text { Type } 2 \\
(\mathrm{MC}, \mathrm{NP}, \mathrm{INF}) \\
(\mathrm{n}=126)\end{array}$ & $\begin{array}{c}p \text {-value } \\
\text { (type } 1 \text { vs 2) }\end{array}$ \\
\hline Tumor size (>5 cm) & 0 & $11(10.3)$ & $21(26.9)$ & $7(21.9)$ & $6(37.5)$ & $11(9.5)$ & $34(27.0)$ & $<.001$ \\
\hline HBV etiology & $5(55.6)$ & $73(68.2)$ & $58(74.4)$ & $21(65.6)$ & $15(93.8)$ & $78(67.2)$ & $94(74.6)$ & .207 \\
\hline Edmondson-Steiner grade III/IV & $4(44.4)$ & $64(59.8)$ & $58(74.4)$ & $25(78.1)$ & $15(93.8)$ & $68(58.6)$ & $98(77.8)$ & .001 \\
\hline Microvascular invasion & $1(11.1)$ & $29(27.1)$ & $34(43.6)$ & $14(43.8)$ & $10(62.5)$ & $30(25.9)$ & $58(46.0)$ & $<.001$ \\
\hline Portal vein invasion & 0 & 0 & $4(5.1)$ & $5(15.6)$ & $8(50.0)$ & 0 & $17(13.55)$ & $<.001$ \\
\hline High T category (pT3 or pT4) & 0 & $1(0.9)$ & $7(9.0)$ & $5(15.6)$ & $7(43.8)$ & $1(0.9)$ & $19(15.1)$ & $<.001$ \\
\hline Cirrhosis in background liver & $6(66.7)$ & $49(46.7)$ & $42(57.5)$ & $15(46.9)$ & $14(87.5)$ & 55 (48.2) & $71(58.1)$ & .118 \\
\hline Serum AFP level >1,000 ng/mL & 0 & $12(13.6)$ & $10(14.7)$ & $6(20.7)$ & $6(37.5)$ & $12(12.6)$ & $22(19.5)$ & .194 \\
\hline Fibrous stroma (> 30\%) & $1(11.1)$ & $14(13.1)$ & $25(32.1)$ & $9(28.1)$ & $6(37.5)$ & $15(12.9)$ & $40(31.7)$ & $<.001$ \\
\hline CK19 positive & $2(22.2)$ & $10(9.3)$ & $20(25.6)$ & $7(21.9)$ & $6(37.5)$ & $12(10.3)$ & $33(26.2)$ & .002 \\
\hline EpCAM positive & $3(33.3)$ & 37 (34.6) & $34(43.6)$ & $19(59.4)$ & $12(75.0)$ & $40(34.5)$ & $65(51.6)$ & .009 \\
\hline UPAR positive & $1(11.1)$ & $16(15.2)$ & $22(28.2)$ & $14(43.8)$ & $12(75.0)$ & $17(14.9)$ & $48(38.1)$ & $<.001$ \\
\hline Ezrin positive & $4(44.4)$ & $34(32.4)$ & $40(51.3)$ & $12(37.5)$ & $7(43.8)$ & 38 (33.3) & $59(46.8)$ & .036 \\
\hline
\end{tabular}

VN, vaguely nodular; EN, expanding nodular; MC, multinodular confluent; NP, nodular with perinodular extension; INF, infiltrative; HBV, hepatitis B virus; AFP, $\alpha$-fetoprotein; CK19, cytokeratin 19; EpCAM, epithelial cell adhesion molecule; UPAR, urokinase plasminogen activator receptor.
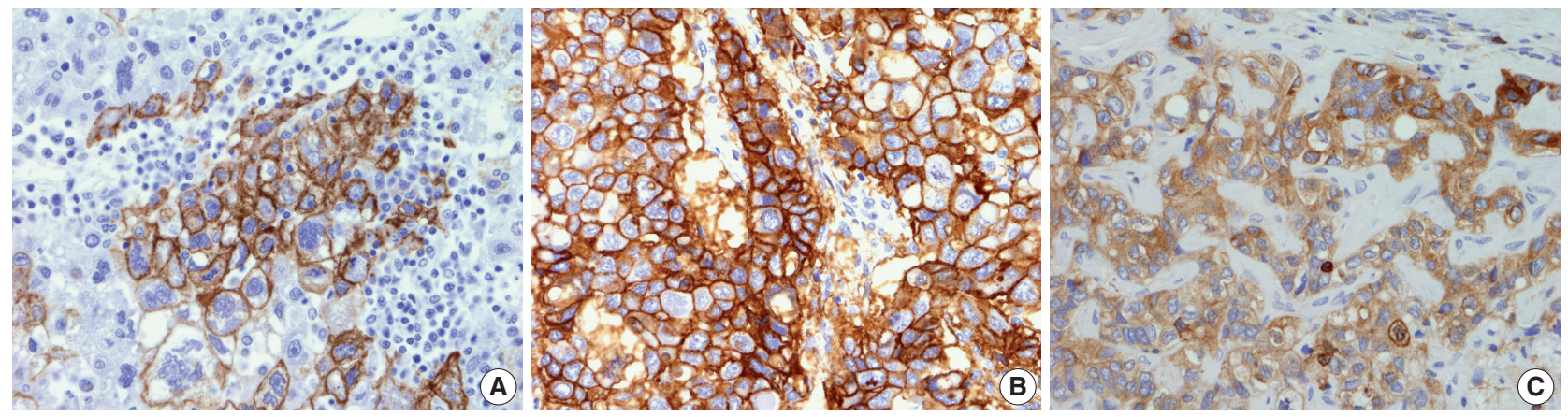

Fig. 2. Immunohistochemical stain results for stemness- and epithelial-mesenchymal transition-related markers: cytokeratin 19 (A), epithelial cell adhesion molecule (B), and ezrin (C). 
Table 3. Survival analysis results

\begin{tabular}{|c|c|c|c|c|}
\hline \multirow[b]{2}{*}{ Variable } & \multicolumn{2}{|c|}{ Overall survival } & \multicolumn{2}{|c|}{ Disease-free survival } \\
\hline & $\begin{array}{c}\text { Hazard ratio } \\
\text { (95\% confidence interval) }\end{array}$ & $p$-value & $\begin{array}{c}\text { Hazard ratio } \\
\text { (95\% confidence interval) }\end{array}$ & $p$-value \\
\hline \multicolumn{5}{|l|}{ Univariate analysis } \\
\hline Gross type (type 1 vs type 2) ${ }^{a}$ & 5.439 (1.583-18.683) & .007 & $1.617(1.092-2.394)$ & .016 \\
\hline Tumor size $(>5 \mathrm{~cm})$ & $1.229(0.407-3.712)$ & .714 & $1.590(1.013-2.495)$ & .044 \\
\hline High E-S grade (III or IV) & $0.636(0.256-1.583)$ & .331 & $1.054(0.694-1.599)$ & .805 \\
\hline Microvascular invasion & $2.178(0.881-5.383)$ & .092 & $1.445(0.977-2.138)$ & .065 \\
\hline Portal vein invasion & $5.311(1.906-14.796)$ & $<.001$ & $1.702(0.858-3.376)$ & .128 \\
\hline High T category (pT3 or pT4) & 5.206 (1.974-13.728) & $<.001$ & $1.795(0.982-3.284)$ & .057 \\
\hline Intratumoral stromal fibrosis & $2.037(0.801-5.179)$ & .135 & $0.973(0.608-1.559)$ & .973 \\
\hline \multicolumn{5}{|l|}{ Multivariate analysis } \\
\hline Gross type (type 1 vs type 2) & $4.118(1.142-14.844)$ & .030 & $1.617(1.092-2.394)$ & .016 \\
\hline Tumor size $(>5 \mathrm{~cm})$ & $0.586(0.166-2.060)$ & .404 & $1.303(0.802-2.115)$ & .285 \\
\hline Microvascular invasion & 1.953 (0.765-4.982) & .161 & $1.300(0.866-1.952)$ & .205 \\
\hline Portal vein invasion & $1.091(0.150-7.931)$ & .931 & $0.954(0.292-3.124)$ & .938 \\
\hline High T category (pT3 or pT4) & $3.173(1.156-8.710)$ & .025 & $1.462(0.500-4.275)$ & .487 \\
\hline
\end{tabular}

E-S grade, Edmondson-Steiner grade.

aType 1: vaguely nodular and expanding nodular types, type 2: multinodular confluent, nodular with perinodular extension and infiltrative types.
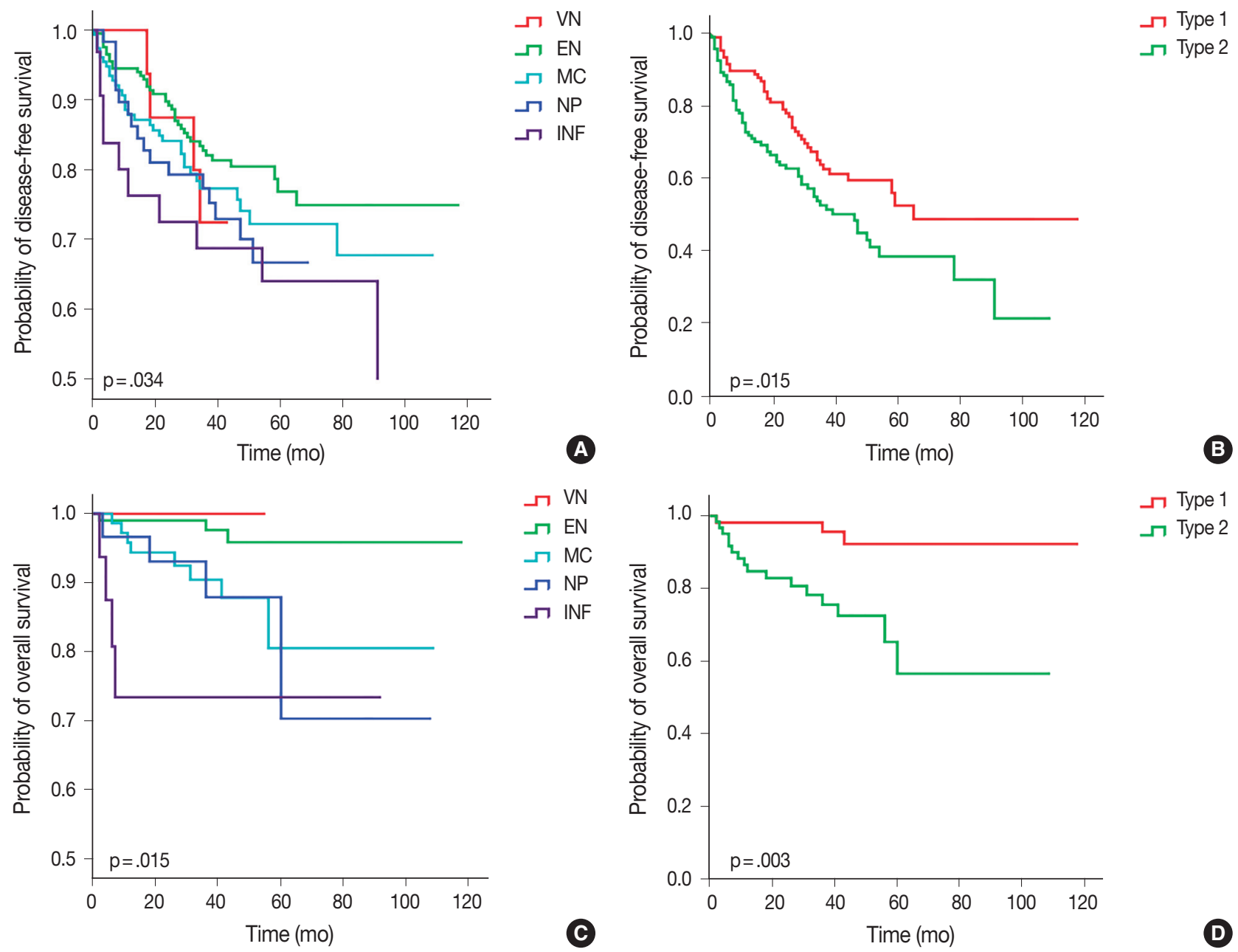

Fig. 3. Kaplan-Meier curves demonstrating differences in disease-free survival and overall survival according to gross classification of hepatocellular carcinoma. (A, B) Disease-free survival. (C, D) Overall survival. VN, vaguely nodular; EN, expanding nodular; MC, multinodular confluent; NP, nodular with perinodular extension; INF, infiltrative. 


\section{Survival analysis results}

Of the five different gross types, the INF type demonstrated the worst overall survival $(\mathrm{p}=.015)$ and disease-free survival $(\mathrm{p}=.034)$ compared to other types (Table 3, Fig. 3). The most favorable outcome was seen for EN and VN types, and the survival curves for $\mathrm{MC}$ and $\mathrm{NP}$ types were in between that of EN and INF type HCCs. When the gross types were simplified into two groups, type 2 HCCs showed significantly decreased diseasefree $(\mathrm{p}=.015)$ and overall survival $(\mathrm{p}=.003)$ compared to type 1 HCCs. Of the other clinicopathological variables, tumor size of larger than $5 \mathrm{~cm}$ was associated with a decreased disease-free survival $(\mathrm{p}=.041)$, portal venous invasion $(\mathrm{p}<.001)$ and higher $\mathrm{pT}$ stage $(\mathrm{p}<.001)$ were associated with decreased overall survival, and microvascular invasion was marginally associated with decreased disease-free and overall survivals although not statistically significant.

Multivariate analysis demonstrated that gross classification (type 1 vs 2) was a significant independent predictor of both overall and disease-free survival, after adjusting for patient sex and age. Type 2 HCCs showed significantly decreased overall survival $(\mathrm{p}=.030$; hazard ratio, $4.118 ; 95 \%$ confidence interval, 1.142 to 14.844$)$ and disease-free survival $(\mathrm{p}=.016$; hazard ratio, 1.617; $95 \%$ confidence interval, 1.092 to 2.394). High pathologic $\mathrm{T}$ stage also remained a significant predictive factor for overall survival $(\mathrm{p}=.025$; hazard ratio, $3.173 ; 95 \%$ confidence interval, 1.156 to 8.710 ).

\section{DISCUSSION}

In this study, we found that INF type HCCs had the worst prognosis out of the five different gross types, and when the HCCs were further grouped into types 1 and 2, gross type 2 HCCs more frequently showed clinicopathological features of aggressive behavior and poor prognosis compared to type 1 HCCs. The gross classification had a strong impact on patient survival; type 2 gross morphology was a significant independent predictor of decreased overall and disease-free survivals on multivariate analysis.

Our findings are similar to the results of a few previous studies. Increased overall and disease-free survival was noted for single nodular type HCCs compared to those with extranodular growth or MC growth patterns. ${ }^{6,9}$ Interestingly, Shimada et al. ${ }^{9}$ analyzed small HCCs $(<3 \mathrm{~cm})$ separately and found similar associations between gross morphology and prognosis, and in another analysis $^{3}$ on huge HCCs $(>10 \mathrm{~cm})$, single nodular type HCCs showed more favorable outcomes compared to non-single nodular
HCCs. Taken together, it could be suggested that the gross classification may be an important predictor of prognosis regardless of tumor size. When tumor size was entered into our multivariate analysis model, we found that gross type 2 was a strong independent predictor of both overall survival and disease-free survival.

The gross classification consists of five different morphological types of HCCs and clear definitions for the different types have been proposed in the guidelines of both the LCSGJ and KLCA. However, in practice, the distinction between NP, MC, and INF type HCCs is not always clear cut, and prone to interobserver variability. On the other hand, EN and VN types (known as "single nodular with distinct margin" and "single nodular with indistinct margin" types in the LCSGJ guidelines, respectively) are relatively easier to discriminate from the other types as they lack the multilobulated irregular contour. If the gross classification is an important prognostic factor for HCC on surgically resected specimens, this could also be implemented in the preoperative evaluation of HCC patients, as the gross features can be determined on preoperative imaging. Therefore, it may be sufficient and more practical to classify HCCs as single nodular types (type 1) and non-single nodular types (type 2) for guiding patient management strategies. Interestingly, Fu et $a l^{4}{ }^{4}$ analyzed the survival of patients with small HCCs $(<5 \mathrm{~cm})$ treated with radiofrequency ablation according to the gross type on imaging, and found that HCCs with single nodular HCCs without extranodular growth or irregular margins were associated with favorable survival.

A subset of HCCs that have morphological features consistent with HCC have been demonstrated to express immunophenotypes associated with "stemness," such as CK19, EpCAM, CD133, and c-kit positivity. These tumors have been associated with higher preoperative serum AFP levels, less frequent fibrous capsule formation, intratumoral fibrous stroma, frequent vascular invasion and poor prognosis compared to typical HCCs that do not express these markers. ${ }^{16,17}$ As expected, we found in this study that larger tumor size, poor histological differentiation, microvascular and portal venous invasion, intratumoral fibrous stroma, "stemness" and EMT-related marker expression and higher T stages were significantly more frequent in type 2 HCCs compared to type 1 HCCs. Therefore, HCCs that have a solitary, well-circumscribed and expansile growth pattern were less likely to exhibit features associated with "stemness." The higher prevalence of CK19 expression in type 2 HCCs has been recently demonstrated by another group.'

EMT refers to the process in which tumor epithelial cells lose their epithelial characteristics (e.g., loss of membranous E-cad- 
herin expression) and acquire mesenchymal features, facilitating tumor invasion and distant metastasis. ${ }^{18}$ This process has been described in HCCs, and HCCs expressing ezrin and UPAR have been associated with poor prognoses. ${ }^{19,20}$ We found in this study that INF type HCCs showed frequent uPAR expression (75\%) compared to other types, especially $\mathrm{VN}$ and EN types which were uPAR positive in $11 \%$ and $15 \%$, respectively. MC and NP types showed uPAR expression frequency intermediate between INF types and EN/VN types. In a previous study from Japan, E-cadherin loss was more frequently seen in single nodular with extranodal growth type and confluent multinodular type HCCs of less than $6 \mathrm{~cm}$ in diameter (which can be translated to NP and MC type HCCs according to the KLCA classification). ${ }^{7}$ Taken together, it could be suggested that invasiveness and metastatic ability of HCCs could be reflected by the gross appearance.

On examining the Kaplan-Meier curves for overall and diseasefree survival according to the five different gross types of our cohort, significant differences in survival were noted between the EN types and INF types. The MC and NP types showed survivals intermediate between the EN and INF types, without significant differences between the two types. Interestingly, while the VN type showed the best overall survival (no HCCrelated deaths), early recurrences were noted for two VN type HCCs (at 17 and 18 months) for the disease-free survival analysis. Although VN type morphology is a characteristic feature of early HCC, ${ }^{15}$ we included all cases that were macroscopically of $\mathrm{VN}$ type regardless of the histological differentiation or tumor size; indeed, poor histological differentiation was noted in $4 / 9$ (44.4\%) cases and 2/9 (22.2\%) cases were larger than $3 \mathrm{~cm}$. Therefore, the VN type in this study does not refer to early HCC, and we grouped $\mathrm{VN}$ and EN types together into type 1 (single nodular) HCCs for analytical purposes.

Although this is not the first report on the clinicopathological significance of the gross classification of HCCs, this is a largescale cohort study of 242 surgically resected solitary HCCs using the definitions in the guidelines of the KLCA, and we also demonstrate for the first time the associations between the different gross types and the expression status of "stemness"- and EMTrelated markers. A limitation of this study is that we excluded multiple HCCs (including multicentricity and intrahepatic metastasis) from the study cohort in order to exclude cases showing multiple gross types in the same liver. This resulted in the lower percentage of cases with higher $\mathrm{pT}$ stage, and the exclusion of pT3a cases using the current AJCC staging system (seventh edition). Nevertheless, we demonstrate that the gross classification of HCCs according to the KLCA guidelines has prognostic value, and that gross type 2 HCCs with non-single nodular patterns are associated with clinicopathological features of aggressive behavior, increased expression of "stemness"- and EMT-related markers and decreased survival. Further validation would be required in independent cohorts and also radio-pathological correlation studies would be needed to validate the utility of the gross classification in HCC patient management.

\section{Conflicts of Interest}

No potential conflict of interest relevant to this article was reported.

\section{Acknowledgments}

This work was supported by grant number 14-2014-012 from the SNUBH Research Fund, and the Basic Science Research Program through NRF funded by the Ministry of Education (NRF2016R1D1A1A09919042).

\section{REFERENCES}

1. Liver Cancer Study Group of Japan. General rules for the clinical and pathological study of primary liver cancer. 2nd ed. Tokyo: Kanehara \& Co., 2003.

2. Kanai T, Hirohashi S, Upton MP, et al. Pathology of small hepatocellular carcinoma: a proposal for a new gross classification. Cancer 1987; 60: 810-9.

3. Choi GH, Han DH, Kim DH, et al. Outcome after curative resection for a huge $(>\mathrm{or}=10 \mathrm{~cm})$ hepatocellular carcinoma and prognostic significance of gross tumor classification. Am J Surg 2009; 198: 693701.

4. Fu X, Mao L, Tang M, et al. Gross classification of solitary small hepatocellular carcinoma on preoperative computed tomography: prognostic significance after radiofrequency ablation. Hepatol Res 2016; 46: 298-305.

5. Gong SC, Cho MY, Lee SW, Kim SH, Kim MY, Baik SK. The meaning of gross tumor type in the aspects of cytokeratin 19 expression and resection margin in patients with hepatocellular carcinoma. J Gastroenterol Hepatol 2016; 31: 206-12.

6. Hui AM, Takayama T, Sano K, et al. Predictive value of gross classification of hepatocellular carcinoma on recurrence and survival after hepatectomy. J Hepatol 2000; 33: 975-9.

7. Inayoshi J, Ichida T, Sugitani S, et al. Gross appearance of hepatocellular carcinoma reflects E-cadherin expression and risk of early recurrence after surgical treatment. J Gastroenterol Hepatol 2003; 18: 673-7. 
8. Murakata A, Tanaka S, Mogushi K, et al. Gene expression signature of the gross morphology in hepatocellular carcinoma. Ann Surg 2011; 253: 94-100.

9. Shimada M, Rikimaru T, Hamatsu T, et al. The role of macroscopic classification in nodular-type hepatocellular carcinoma. Am J Surg 2001; 182: 177-82.

10. Trevisani F, Caraceni P, Bernardi M, et al. Gross pathologic types of hepatocellular carcinoma in Italian patients: relationship with demographic, environmental, and clinical factors. Cancer 1993; 72: 1557-63.

11. Tsujita E, Yamashita Y, Takeishi K, et al. The clinicopathological impact of gross classification on solitary small hepatocellular carcinoma. Hepatogastroenterology 2013; 60: 1726-30.

12. A new prognostic system for hepatocellular carcinoma: a retrospective study of 435 patients: the Cancer of the Liver Italian Program (CLIP) investigators. Hepatology 1998; 28: 751-5.

13. Jang JY, Lee JS, Kim HJ, et al. The general rules for the study of primary liver cancer. J Liver Cancer 2017; 17: 19-44.

14. Kurogi M, Nakashima O, Miyaaki H, Fujimoto M, Kojiro M. Clinicopathological study of scirrhous hepatocellular carcinoma. J Gastroenterol Hepatol 2006; 21: 1470-7.

15. International Consensus Group for Hepatocellular Neoplasia.
Pathologic diagnosis of early hepatocellular carcinoma: a report of the international consensus group for hepatocellular neoplasia. Hepatology 2009; 49: 658-64.

16. Kim H, Choi GH, Na DC, et al. Human hepatocellular carcinomas with "stemness"-related marker expression: keratin 19 expression and a poor prognosis. Hepatology 2011; 54: 1707-17.

17. Kim H, Park YN. Hepatocellular carcinomas expressing 'stemness'related markers: clinicopathological characteristics. Dig Dis 2014; 32: 778-85.

18. Lee JM, Dedhar S, Kalluri R, Thompson EW. The epithelial-mesenchymal transition: new insights in signaling, development, and disease. J Cell Biol 2006; 172: 973-81.

19. Okamura D, Ohtsuka M, Kimura F, et al. Ezrin expression is associated with hepatocellular carcinoma possibly derived from progenitor cells and early recurrence after surgical resection. Mod Pathol 2008; 21: 847-55

20. Zhou L, Hayashi Y, Itoh T, Wang W, Rui J, Itoh H. Expression of urokinase-type plasminogen activator, urokinase-type plasminogen activator receptor, and plasminogen activator inhibitor- 1 and -2 in hepatocellular carcinoma. Pathol Int 2000; 50: 392-7. 